\title{
Control of Mycobacterium bovis infection in two sika deer herds in Ireland
}

\author{
Tom Partridge ${ }^{1}$, Dónal Toolan², John Egan ${ }^{3}$ and Simon More ${ }^{4}$ \\ 1 Department of Agriculture Fisheries \& Food, District Veterinary Office, Waterford, Co. Waterford, Ireland \\ 2 Department of Agriculture Fisheries \& Food, Kilkenny Regional Veterinary Laboratory, Hebron Road, Leggatsrath, Co. Kilkenny, Ireland \\ 3 Department of Agriculture Fisheries \& Food, Central Veterinary Research Laboratory, Backweston Campus, Co. Kildare, Ireland \\ 4 Centre for Veterinary Epidemiology and Risk Analysis, University College Dublin, Belfield, Dublin 4, Ireland
}

\author{
Corresponding author: \\ Tom Partridge \\ Department of Agriculture Fisheries \& Food, District Veterinary Office, Waterford, Co. Waterford, Ireland \\ Tel: 0035351301600 \\ Email: tom.partridge@agriculture.gov.ie
}

\begin{abstract}
In a number of countries, tuberculosis (due to infection with Mycobacterium bovis) is a significant health problem of captive deer. This paper describes outbreaks of bovine tuberculosis in sika deer (Cervus nippon) on two farms in Ireland and the methods used to control the disease. On Farm A, infection was first detected during 1993. The infection was eradicated using a programme of test and removal, in association with segregation of young animals. A second outbreak (also due to infection with $M$. bovis, but a different RFLP profile) was detected in 2002. In the latter outbreak, infection was particularly prevalent in two groups of young deer. $M$. bovis with the same RFLP profile was also isolated in a badger found dead on the farm. Control was achieved by test and removal in association with herd management changes. In Herd B, infection was first detected in 1995, and subsequently eradicated using test and removal alone. In Herd A, re-infection remains an ongoing risk. Control rather than eradication of infection may more realistic in the short- to medium-term.
\end{abstract}

Key words: Mycobacterium, bovis, tuberculosis, sika, deer, Cervus, nippon

Irish Veterinary Journal

\section{Introduction}

Tuberculosis (TB) in deer, caused by Mycobacterium bovis, has been diagnosed in every country where deer are managed as a domestic species (Griffin and Buchan, 1994). A range of strategies has been developed by national agencies to deal with this problem, generally in accordance with local control programmes for tuberculosis in cattle. Red, fallow and sika deer are farmed in Ireland.

Approximately 500 herds were present in 1995, but 200-250 farms (averaging 50-60 breeding deer per herd) remained in 2007, reflecting the poor economic viability of deer farming. The reduction in the number of producers, the enlargement of herds and the increase in the number of processors have helped to stabilise the industry and make it more profitable for those remaining. Most venison produced in Ireland is exported.

Wild red, fallow and sika deer are also found in Ireland. Fallow are the most common species and are found in virtually every county. The prevalence of TB in wild deer populations in Ireland is unknown, although $4 \%$ was recorded in a limited survey of 130 wild red and sika deer in the early 1980s (Dodd, 1984). The prevalence of TB in farmed deer is also unknown but the disease is a major problem in some herds. TB in deer is a notifiable disease in Ireland under the Diseases of Animals Order (1992). Trade in live deer within the European Community is subject to certification of freedom from tuberculosis and brucellosis under the EC's Trade in Animals and Animal Semen, Ova and Embryos Regulations (1996). Although there is no legal obligation for Irish deer farmers to test their herds for $\mathrm{TB}$, deer are subjected to a post mortem examination at slaughter. Standards for post mortem examination were described in European Communities (rabbit and farmed game meat) Regulations, 1995, superseded by Regulation (EC) No 854/2004.

This paper describes outbreaks of bovine tuberculosis in sika deer (Cervus nippon) on two farms in southeast Ireland and the methods used to control the disease. Some insights into the application of the single intradermal comparative tuberculin test (SICTT) in sika deer are also described. 


\section{Materials and methods \\ Case farms}

The two case farms, each farming deer as the sole farm enterprise, were located in Ireland. Farm A consisted of 30 hectares, of which 28 hectares were fenced for the purpose of deer farming and were subdivided into three sections by internal fences (Figure 1). Similarly, 12 of 16 hectares of Farm B were fenced and subdivided into six sections. The perimeter and internal fencing on both farms were made of wire mesh $1.8 \mathrm{~m}$ high.

On both farms, calves were normally weaned during November and moved to a separate section on the farm. Apple pulp was the main supplementary winter feed, complemented with hay or dried beet nuts and was fed on a daily basis, usually in the morning, and was deposited on the ground. Deer were out-wintered, with adequate cover and shelter for the animals in adverse weather conditions. Farm A was surrounded by beef and dairy establishments and was also contiguous to a sheep flock. TB breakdowns had occurred in cattle herds around Farm A, largely coinciding with the 1994 and 2002 breakdowns in deer. Farm B was contiguous to forest and two suckler herds. The area had a lower prevalence of TB compared to the area around Farm $\mathrm{A}$ and the pattern of breakdowns was different in these cattle herds. Natural barriers plus adequate fencing were sufficient to prevent contact, either direct or indirect, between deer on both farms and cattle on contiguous farms.

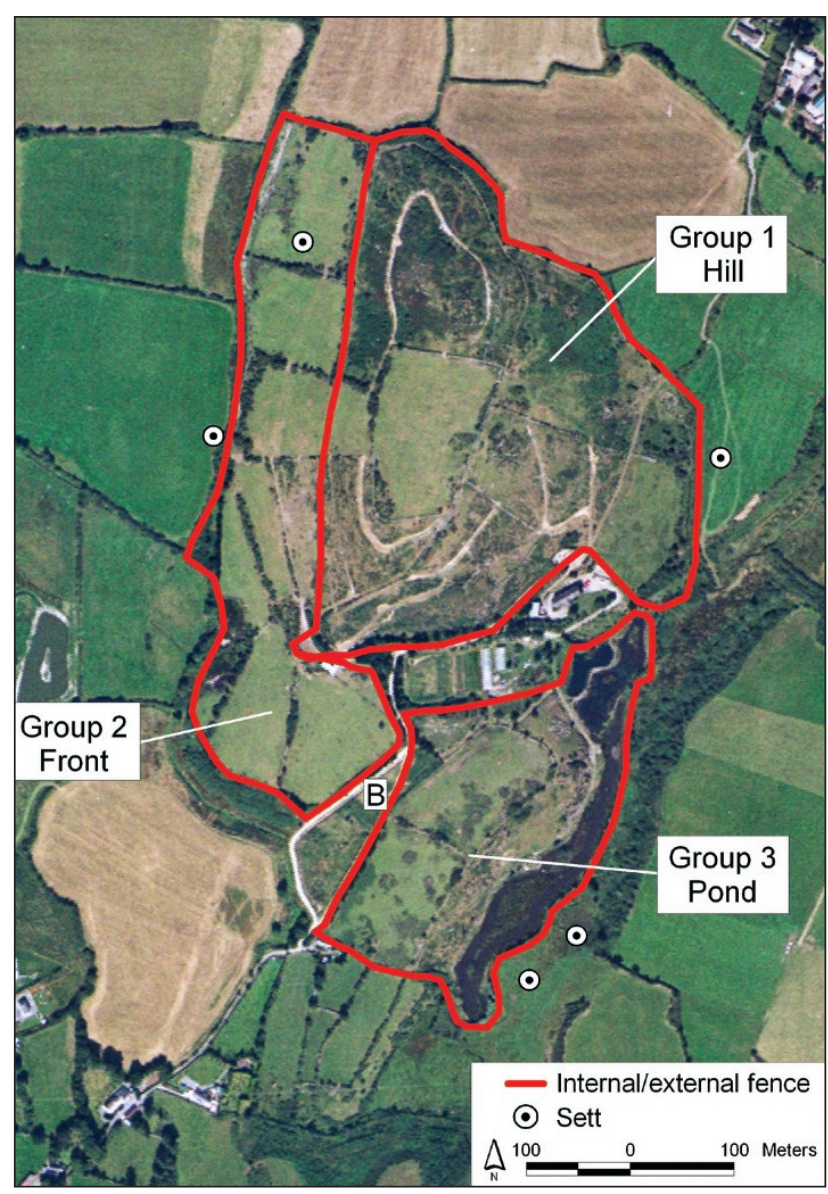

Figure 1: Map and aerial photograph of Farm A, showing the location of deer groupings (red outline), the site where a tuberculous badger was found in February 2002 (B), and the location of badger setts (white).

\section{Diagnostic methods}

On each farm, tuberculin (TB) testing was conducted using the SICTT. On the two case farms, good handling facilities were available, enabling these tests to be conducted with precision. The deer were tested indoors, and artificial lighting was used on dull days. Sika deer, due to their small size and quiet temperament, were easy to handle. The same tester initiated and completed each SICTT to ensure consistency.

On each farm, the SICTT was conducted using the following methodology. A coarse electric shears (Liscop Super 3000-Type 1300-2-TD) was used to remove heavy hair from one side of the neck and then a fine electric shears (Oyster no. 80, size 40) was used to prepare two injection sites. Each injection site was approximately $45 \mathrm{~mm}$ square, and both sites were located $70-80 \mathrm{~mm}$ apart, in the middle third of the neck. Each site was palpated and unusual features (lumps etc.) noted. A fold of skin was lifted at each site, between the thumb and forefinger and measured using digital calipers. All measurements were recorded. Then, at the centre of the upper and lower sites, respectively, $0.1 \mathrm{ml}$ of avian tuberculin PPD $(25,000 \mathrm{IU} / \mathrm{ml})$ and bovine tuberculin PPD (30,000 Ph. Eur. U/ml, each from the Institute for Animal Science and Health, Lelystad, the Netherlands) were injected intradermally using a single-dose $1 \mathrm{ml}$ insulin syringe and 26-gauge, 3/8 inch needle. Needles were disinfected before each injection using methylated spirits. When the intradermal injection was properly executed, a slight resistance was evident and a 'bleb', which tended to spread, was observed to form. A lack of resistance indicated the injection was subcutaneous. $72 \pm 4$ hours after injection, the injection sites were examined and measured to the nearest $0.1 \mathrm{~mm}$. Precise measurements were required as the changes in skin thickness were often minimal. The skin reaction (the difference between the skin measurements at 0 and 72 hours) was interpreted according to the schema described in Figure 2. A severe interpretation was applied where TB infection in a herd had recently been established; otherwise a standard interpretation was made.

Throughout the testing programme in both herds, test-positive animals were euthanased and a detailed gross post mortem examination was conducted, by the Veterinary Laboratory Service, at the Central Veterinary Research Laboratory (CVRL; Abbotstown, Co. Dublin) or the Kilkenny Regional Veterinary Laboratory (KRVL; Kilkenny, Co. Kilkenny). The retropharyngeal, submaxillary, bronchial, mediastinal, parotid and mesenteric lymph nodes were examined, as were the liver and lungs. A large percentage of tissue samples showing lesions suspicious of tuberculosis were subjected to histopathological examination and/or culture. Normal tissues from a random subset of these animals were also subjected to culture and histopathological examination. Samples for culture were processed as outlined by Quigley et al. (1997) and cultured as outlined by Collins et al. (1997). Isolates were strain typed using the RFLP method as outlined by Costello et al. (1999). 

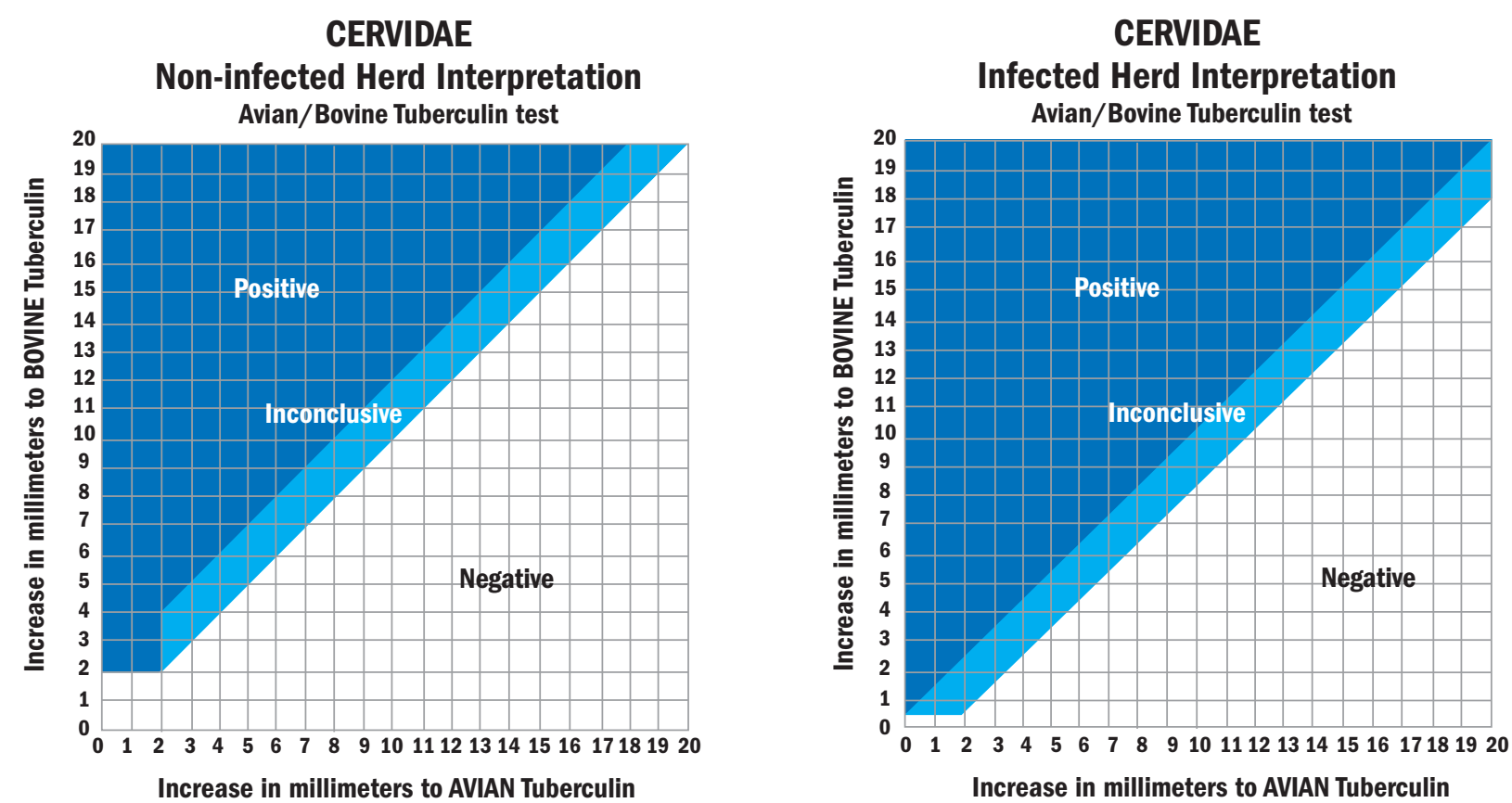

Figure 2: Charts used for the standard (left) and severe (right) interpretation of the single intradermal cervical tuberculin test (SICTT) in deer.

Throughout the study period, all animals submitted for routine slaughter were subjected to post mortem examination (PME) including examination of the lungs, livers and the lymphatic glands in the head, thorax and abdomen. On most occasions a number of additional veterinary staff were available to undertake a more enhanced PME which allowed for additional detailed slicing of the organs and tissues above for evidence of tuberculosis in 406 of the 611 animals slaughtered between 1998 and 2004. Suspicious lesions were collected for histological and cultural examination.

\section{Results}

\section{Herd A (Deer)}

Infection with bovine tuberculosis was first detected in Herd A (the deer on Farm A) during routine post mortem examination of deer slaughtered in the factory in 1993. TB testing commenced during spring 1994. Testing was conducted twice annually in spring and autumn, except during 1998, 2001, 2005 and 2006 when only the spring test was undertaken (Table 1).

Skin test reactors were detected from 1994 to 1997. During 1998, due to competing work commitments, only a partial herd test was conducted. In that year, 87 animals were examined during enhanced factory surveillance in October. Three animals had visible lesions in the mesenteric lymph nodes; these lesions were histologically consistent with tuberculosis, but were negative on culture. A female culled in early 1999 because of poor condition showed gross tuberculous lesions in the lung and $M$. bovis was isolated on culture. No reactors were found during 2000 (Table 1). Although no herd test was undertaken during the period of the FMD crisis in 2001, 55 deer were tested prior to sale, and tested negative.

In 1995, to manage and control the initial TB breakdown, young test-negative animals (animals less than two years and SICCT-negative) were separated from the older males and females, and subsequently managed as a separate herd on the pond side of the farm (Figure 1). These animals, which formed the basis of Group 3 (see below), never again came into contact with the older stock on the hill. At the start of 2002, Herd A deer were managed as three separate groups, including Groups 1 (original herd 1995), 2 (calves and yearlings) and 3 (animals derived from the new herd established in 1994). Group 3 had no physical contact with either Groups 1 or 2, whereas Groups 1 and 2 were separated by a wire mesh fence (Figure 1). In late winter/ early spring 2002, 60 2001-born calves to females from Group 3 had been moved to join 74 Group 2 yearlings, two weeks prior to the commencement of testing.

In February 2002, a female deer, from Group 3, with signs of pneumonia was presented for clinical examination. TB was suspected and the deer was humanely killed. Gross lesions of tuberculosis were found and M. bovis was subsequently cultured. The entire herd was then tested during spring 2002, and the results indicated severe TB infection (Tables 1 and 2). At the first TB test on Group 2 animals (during March 2002), 20 (of 60; 33\%) calves and 21 (of 74; 28.4\%) yearlings were skin test positive. At the first TB test on animals in Group 3 (during April 2002), 12 (of 28; 42.9\%) eight year-old females, 22 (of 42; 52\%) three-year-old females, 12 (of 22; $54.5 \%$ ) yearlings and two (of $5 ; 40.0 \%$ ) stags were positive. On a second test of Group 2 (during May 2002) three yearlings and eight calves were positive. At the first TB test on animals in Group 1 (during April 2002), 17 (of 301, 5.6\%) were positive, including 11 adults and six calves (Table 2).

During 2002-2006, 146 test reactors were examined at post mortem, and 91 (62.3\%) had gross lesions. From the 91 gross lesions, 44 (48.4\%) were examined histologically and $41(93.2 \%)$ were positive. Of the 41 histologically positive, 18 were submitted for culture and $15(83.3 \%)$ were positive 
Table 1: Tuberculosis (TB) testing history of Herds A and B during 1994 to 2007: number tested and the number and percentage positive

\begin{tabular}{|c|c|c|c|c|c|}
\hline \multirow[t]{2}{*}{ Year } & \multirow[t]{2}{*}{ Season } & \multicolumn{2}{|l|}{ Herd A } & \multicolumn{2}{|l|}{ Herd B } \\
\hline & & $\begin{array}{l}\text { No. } \\
\text { tested }\end{array}$ & $\begin{array}{l}\text { No. } \\
\text { positive. } \\
(\%)\end{array}$ & No. tested & $\begin{array}{l}\text { No. } \\
\text { positive } \\
(\%)\end{array}$ \\
\hline \multirow[t]{2}{*}{1994} & Spring & 150 & 20 (13.0) & & \\
\hline & Autumn & 206 & $28(13.6)$ & & \\
\hline \multirow[t]{2}{*}{1995} & Spring & 318 & 74 (22.3) & & \\
\hline & Autumn & 426 & $5(1.2)$ & & \\
\hline \multirow[t]{2}{*}{1996} & Spring & 318 & $5(1.6)$ & 178 & 18 (10.1) \\
\hline & Autumn & 305 & $3(1.0)$ & 115 & $7(6.0)$ \\
\hline \multirow[t]{2}{*}{1997} & Spring & 363 & $2(0.6)$ & 145 & 0 \\
\hline & Autumn & 387 & 0 & nd & \\
\hline \multirow[t]{2}{*}{1998} & Spring & 82 & 0 & 137 & 0 \\
\hline & Autumn & nd & & nd & \\
\hline \multirow[t]{2}{*}{1999} & Spring & 452 & 0 & 206 & 0 \\
\hline & Autumn & 82 & 0 & nd & \\
\hline \multirow[t]{2}{*}{2000} & Spring & 312 & 0 & nd & \\
\hline & Autumn & 104 & 0 & nd & \\
\hline \multirow{2}{*}{2001} & Spring & 55 & 0 & nd & \\
\hline & Autumn & nd & & nd & \\
\hline \multirow[t]{2}{*}{2002} & Spring & $625^{a}$ & $\begin{array}{l}117 \\
(18.7)\end{array}$ & nd & \\
\hline & Autumn & 142 & $16(11.0)$ & nd & \\
\hline \multirow[t]{2}{*}{2003} & Spring & 399 & $7(1.8)$ & 190 & 0 \\
\hline & Autumn & 343 & $1(0.3 \%)$ & nd & \\
\hline \multirow[t]{2}{*}{2004} & Spring & 389 & 0 & nd & \\
\hline & Autumn & 275 & 0 & nd & \\
\hline \multirow[t]{2}{*}{2005} & Spring & 277 & $2(0.7)$ & 201 & 0 \\
\hline & Autumn & nd & & nd & \\
\hline \multirow[t]{2}{*}{2006} & Spring & 336 & $3(0.9)$ & nd & \\
\hline & Autumn & nd & nd & nd & \\
\hline 2007 & Spring & 339 & 0 & nd & \\
\hline \multicolumn{6}{|c|}{$\begin{array}{l}\text { a: This number includes a retest on Group } 2 \text { during spring } 2002 \\
\text { (Table 2). } \\
\text { In Herd A, severe interpretation of the SICTT results was applied } \\
\text { throughout the testing programme, whereas in Herd B, severe } \\
\text { interpretation was only applied in } 1996 / 97 .\end{array}$} \\
\hline
\end{tabular}

for M. bovis, one (5.6\%) was positive for M. avium and two $(11.1 \%)$ cultured negative. Microbiological examination was also conducted on $36(65.4 \%$ of the 55$)$ animals without gross lesions, and 11 (30.6\%) were positive for M. bovis.

One animal with gross lesions was negative on histology but positive on culture. During 1996 to 2006, 71 animals were submitted for detailed examination, after being culled (64) or found dead (7). Of these, five had gross and histopathological lesions consistent with TB, and from four of the five, cultures were positive for M. bovis (Table 3). During 1998 to 2004, 406 of 611 deer sent for routine slaughter to the abattoir were subjected to an enhanced post mortem examination. Three of the 406 showed mesenteric lesions which were positive on histology but negative on culture. The remaining 403 showed no evidence of gross TB.
Table 2: Tuberculin testing results for Herd A during 2002 and spring 2003: number tested, number reactors, percentage reactors, number reactors with visible lesions at post-mortem, percentage with visible lesions at post mortem, by test number and Group

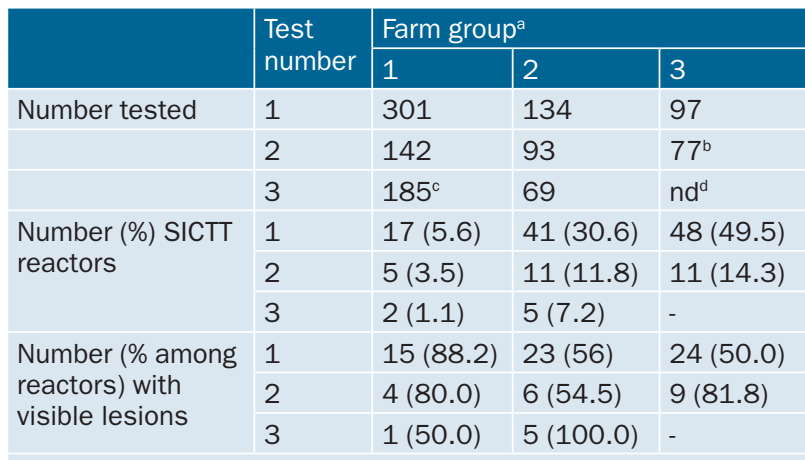

a: Group 1 consisted of adults, yearlings and calves, Group 2 calves and yearlings, and Group 3 adults, yearlings and calves. b: A test in 2002 (on September 23) was conducted on all deer suitable for slaughter from Groups 2 and 3 . Eleven reactors, from both groups, were detected, including nine with gross lesions.

The test negative animals, from both groups, were sent direct to slaughter and no gross lesions were detected.

c: Included calves in Group 1 that had not previously been tested and also the balance of Group 1 not tested in test 2 .

d: Not done. Remaining Group 3 animals were slaughtered after test 2.

\section{Herd A (Badgers)}

In February 2002, a badger with a suppurating neck wound was found dead in close proximity to deer in Groups 2 and 3 on Farm A (Figure 1). Gross lesions typical of tuberculosis were found, and $M$. bovis was cultured from the neck wound, lung tissue and head glands. Although badgers regularly moved onto Farm A, as evidenced by breaches in the perimeter fencing and the presence of badger hairs on the wire fence at these locations, the only badger sett on the farm was unoccupied throughout 1994 to 2007 (Figure 1). Badger removal operations were conducted in the proximity of Farm A during 1996/97 and 2002/03. In 1996/97, 16 badgers were caught at the boundary fence within the farm boundaries, including seven animals with histopathological evidence (six also had gross evidence) of TB infection. Two of the seven samples were sent for culture and were positive for M. bovis. During 2002/03, 17 badgers were caught, including three on the farm and 14 at four badger setts adjacent to the farm boundary (Figure 1). Ten of 17 of these animals had histopathological evidence (eight also had gross evidence) of TB infection respectively.

\section{Herd A (M. bovis strain types isolated)}

The RFLP profile of the $M$. bovis isolates from deer during the breakdowns in 1994 and 2002/03 were different (Table 4). No isolates from badgers removed during the first breakdown were available for strain typing. In 2002/03, the $M$. bovis isolates from deer and a badger shared a common RFLP profile.

\section{Herd B}

Herd B (the deer on Farm B) originated from Herd A, following the purchase of 43 females and one stag in 1992. A further two stags were introduced from Killarney in 1993. Infection was first detected in Herd B following an 
Table 3: Post-mortem, histology and culture results of Herd A animals culled (60 animals) or found dead (11) during 1996 to 2006

\begin{tabular}{|c|c|c|c|c|}
\hline \multirow[t]{2}{*}{ Year } & \multicolumn{2}{|c|}{ Animals culled or found dead } & \multicolumn{2}{|c|}{ No confirmed positive } \\
\hline & Total & $\begin{array}{l}\text { No. with visible lesion } \\
\text { at post-mortem }\end{array}$ & Histology & Culture \\
\hline 1996 & 2 & & & \\
\hline 1997 & 3 & & & \\
\hline 1998 & 1 & & & \\
\hline 1999 & 4 & 1 & 1 & 1 \\
\hline 2000 & 8 & & & \\
\hline 2001 & 8 & & & \\
\hline 2002 & 8 & 2 & 2 & 2 \\
\hline 2003 & 16 & & & \\
\hline 2004 & 5 & & & \\
\hline 2005 & 14 & 1 & 1 & 0 \\
\hline 2006 & 2 & 1 & 1 & 1 \\
\hline Total & 71 & 5 & 5 & 4 \\
\hline
\end{tabular}

a: The culls and dead deer were mainly older females Includes single deer diagnosed with TB pneumonia in early 2002 that led to the testing of the herd.

b: Of the $66 \mathrm{NVL}$ (no visible lesion on gross examination) deer, samples from 18 were cultured. All were negative.

on-farm death in 1995, and testing commenced in spring 1996. There were 18 and seven reactors at the tests in spring and autumn 1996, respectively (Table 1). Eighteen of the 25 reactors were part of the original 43 females purchased from Farm A in 1992. Yearly testing continued during 1997, 1998, 1999 and then again in 2003 and 2005, in response to the TB outbreak on Farm A in 2002. Stags had been purchased from Farm A by Farm B shortly before that outbreak. However, there was no further evidence of infection, either during field testing (Table 1) or enhanced factory surveillance. Further, no TB was detected in the few animals subsequently found dead on-farm. A badger removal programme was not introduced on Farm B due to the rapid clearance of TB from the herd.

\section{Discussion}

Tuberculosis is recognised as a very important health problem of captive deer in many countries, including Japan (Yoshikawa et al., 1994), New Zealand (Griffin et al., 1998) and the United Kingdom (Fleetwood et al., 1988). White-tailed deer are also an important wildlife reservoir of bovine tuberculosis in the USA (O'Brien et al., 2006). Several detailed reviews of TB in deer are available (Clifton-Hadley and Wilesmith, 1991; Griffin and Mackintosh, 2000) and these also underline the difficulties associated with eradicating TB infection from deer herds. The present study highlights the challenges associated with TB control and eradication on two deer farms in Ireland. Progress with the control and eradication of infection varied in both herds, most likely accounted for by the differing epidemiological picture in both herds. TB was rapidly eradicated from Herd $\mathrm{B}$ where infection was focused primarily in a group of older animals purchased from Herd A some years previously. Similarly, the 1994 outbreak in Herd A which was primarily focused in older animals, was successfully controlled and eradicated over the subsequent
Table 4: Restriction fragment length polymorphism (RFLP) typing of Mycobacterium bovis strains isolated from deer and a badger during two TB breakdowns in Herd A during 1994 and 2002

\begin{tabular}{|c|c|c|c|c|c|}
\hline \multirow[t]{2}{*}{ Year } & \multicolumn{3}{|c|}{ RFLP analysis ${ }^{a}$} & \multicolumn{2}{|c|}{ Number of isolates from: } \\
\hline & IS6110 & PGRS & DR & Deer & Badgers \\
\hline During 1994 & A1 & D5 & $A$ & 3 & \\
\hline During 2002 & A1 & D8 & A & 4 & 1 \\
\hline
\end{tabular}

four years, using a test and slaughter policy. In contrast, however, the 2002 outbreak in Herd A proved more difficult to eradicate and, based on current progress and noting that further testing will be required, it has taken at least five years to clear infection. Herd A had a clear test in spring 2007. This outbreak, unlike the earlier one, showed that the prevalence of disease in younger animals was higher, with the tests in spring 2002 identifying 117 reactors of which $70(60 \%)$ were less than two years old. (Group 2, 30.6\% positive in March 2002; Group 3, 49.5\% positive in April 2002). In contrast, during the 1994 outbreak the prevalence in younger animals was lower. Testing in 1994/95 produced 122 reactors, of which $40(32.7 \%)$ were less than two years old. The remaining reactors varied in age from 2 to 14 years. Griffin et al., (1998) had also observed that eradication efforts were less effective in herds with wellestablished infection such as herds with more than $30 \%$ of animals infected at the index test and high infection levels among younger animals.

The SICTT was used as the primary method of detecting infected animals on both case farms. Disease eradication was effectively achieved in the three outbreaks through the detection and removal of reactor animals in Herd $\mathrm{B}$ and with additional control measures in Herd A. In our experience, the test proved highly effective in removing infected animals as highlighted by the high level of correlation between the results of the SICTT and PME findings. The proportion of test-positive deer where infection was confirmed was approximately $60 \%$. This figure was an under-estimate because the culturing, especially of the NVL reactors (i.e., those with no visible lesion on gross examination), was not exhaustive. Bearing in mind that not all animals were subjected to extensive microbiological examination for $M$. bovis at PME, it was not possible to determine sensitivity to the SICCT. It has been reported that the performance of the comparative tuberculin test under natural field conditions is extremely variable, varying between $31 \%$ (Griffiths, 1989) and 90\% (Stuart et al., 1988). High levels of sensitivity (91.4\%) were achieved in experimentally infected deer under controlled conditions (Corrin et al., 1993). It has been reported that when the tuberculin test is used in association with other measures, such as quarantine and careful management, the incidence of tuberculosis can be reduced to low levels or eliminated (O’ Reilly and Daborn, 1995).

The SICTT in deer is not without practical and technical difficulties. Herd tests cannot be carried out during the calving (mid May to early July) and rutting (September and October) seasons. Consequently, on the case farms testing 
of infection in Herd A. Following the skin test, there is desensitisation (suppression of skin reactivity) to subsequent SICTT tests, which is still present 60 days following the previous test (Corrin et al., 1993). In this study, testing intervals were always greater than 60 days, generally closer to 90 days; and in some cases the intervals were longer. In sika deer, double skin thickness varies between $1.3 \mathrm{~mm}$ in young deer to $11 \mathrm{~mm}$ in stags. Therefore, considerable care is needed throughout testing, particularly in thin-skinned animals, to ensure that tuberculin is injected intradermally and not subcutaneously. In our experience, the testing procedure needs to be substantially more rigorous in deer than cattle, demanding significantly more diligence from the tester and handlers alike. The relative cost of tuberculin testing is greater in deer than cattle, due to the slow rate of testing and lower monetary value of individual animals, particularly for fallow and sika deer. This has negative implications for the economics of TB eradication in the deer industry.

The source of the first TB outbreak on Farm A in 1994 remains unknown. Although there was a history of periodic TB breakdowns among neighbouring cattle herds, which largely coincided with the breakdowns on Farm A, there was no contact between deer and cattle along the boundary fences. In the second outbreak on Farm A, it is possible to rule out residual infection as the source, given the differing RFLP profile of $M$. bovis isolates in the two outbreaks. In the 2002 outbreak, the same strain of M. bovis was found in deer and a badger, which highlights the potential for badger involvement. Although the RFLP result does not prove that the badger was responsible for transmission to the deer, the badger had a bite wound from which M. bovis was recovered. That bite wound was most likely inflicted by an infected badger. Recent work has highlighted the importance of badgers in the epidemiology of tuberculosis in Irish cattle herds (Griffin et al., 2005). In New Zealand, it is hypothesised that the principal mode of transmission of TB from dying tuberculous possum to deer is via the oral route or droplet inhalation, with the more dominant and inquisitive deer being the first to become infected (Lugton et al., 1997). In this breakdown, winter feeding may have played a role in the transmission of infection. Apple pulp was fed on the ground on a daily basis, which may have facilitated contact among deer and between badger and deer over a sustained period. In 2002, the prevalence of TB was considerably higher in Groups 2 and 3 (in fields close to where a dead infected badger had been found) than Group 1. During both Farm A breakdowns, the TB prevalence among badgers was high.

It is likely that infected badgers continue to be present in and around Farm A. Therefore, if badgers were the source of the second outbreak, reinfection from that source remains an ongoing risk.

\section{Acknowledgements}

We thank the laboratory staff in Kilkenny RVL, particularly Pat Kelleher and Philip Jones. We also thank Eamonn Costello, Orla Flynn, Frances Quigley and Eddie
Weavers in the CVRL; Leigh Corner, Tracy Clegg and Dan Collins from CVERA at UCD and Ian O'Boyle from the District Veterinary Office (DVO) in Waterford for his help and advice. Thanks also to the wildlife unit in Waterford DVO. Finally, we thank the herd owners and their families for their assistance and cooperation, without which this investigation would not have been possible.

\section{References}

Clifton-Hadley, R. S. and Wilesmith, J. W. (1991). Tuberculosis in deer: a review. Veterinary Record 129: 5-12.

Collins, C.H., Grange, J.M. and Yates, M.D. (1997). Cultural methods. In: Tuberculosis Bacteriology, Organisation and Practice. 2nd edn. Oxford, Butterworth-Heinemann. pp 57-68.

Corrin, K.C., Carter, C.E., Kissling, R.C. and de Lisle, G.W. (1993). An evaluation of the comparative tuberculin skin test for detecting tuberculosis in farmed deer. New Zealand Veterinary Journal 41: 12-20. Costello, E. O'Grady, D. Flynn, O., O’Brien, R. Rogers, M., Quigley, F., Egan J. and Griffin. J. (1999). A study of RFLP analysis and spoligotyping for the epidemiological investigation of Mycobacterium bovis infection. Journal of Clinical Microbiology 37: 3217-3222.

Dodd, K., (1984). Tuberculosis in free living deer. Veterinary Record 115: 592-593.

Fleetwood, A. J., Stuart, F. A., Bode, R. and Sutton, J. P. (1988).

Tuberculosis in deer. Veterinary Record 123: 279-280.

Griffin, J.F.T., Bissett, B., Rodgers, C.R. and MacKintosh, C.G. (1998).

Uncontrollable spread of tuberculosis within a deer herd. Proceedings of the Deer Branch of the New Zealand Veterinary Association 15: 227-34.

Griffiths, L. M (1989). Experiences with skin tests in the field. Proc. Vet Deer Society. 3: 19-29.

Griffin, J.F.T. and Buchan, G.S. (1994). Aetiology, pathogenesis and diagnosis of Mycobacterium bovis in deer. Veterinary Microbiology 40: 193205

Griffin, J.F.T. and Mackintosh, C.G. (2000). Diseases in deer: Perceptions, Problems and Progress. The Veterinary Journal 160: 202-219.

Griffin, J. M., Williams, D. H., Kelly, G. E., Clegg, T. A., O’Boyle, I., Collins, J. D. and More, S. J., (2005). The impact of badger removal on the control of tuberculosis in cattle herds in Ireland. Preventive Veterinary Medicine 67: 237-266.

Lugton, I.W., Wilson, P.R., Morris, R.S. and de Lisle. G.W. (1997).

Natural infection of red deer with bovine tuberculosis. New Zealand Veterinary Journal 45: 19-26.

O'Brien, D. J., Schmitt, S. M., Fitzgerald, S. D., Berry, D. E. and Hickling, G. J. (2006). Managing the wildlife reservoir of Mycobacterium bovis: the Michigan, USA, experience. Veterinary Microbiology 112: 313-323.

O'Reilly L. M and Daborn, C. J. (1995). The epidemiology of Mycobacterium bovis infections in animals and man: a review. Tubercule and Lung Disease, 76: Supplement 1, 1-46.

Quigley, F.C., Costello, E., Flynn, O., Gogarty, A., Mc.Guirk, J., Murphy, A. and Egan J. (1997). Isolation of mycobacteria from lymph node lesions in deer in the Republic of Ireland. Veterinary Record 141: 516-518. Stuart, F.A., Manser, P.A. and McIntosh, F.G. (1988). Tuberculosis in imported red deer. Veterinary Record 122: 508-511.

Yoshikawa, H., Fujiwara, T., Seo, H., Oyamada, T., Ogasawara,

T., Tubaki, S., Saitoh, H. and Yoshikawa, T. (1994). An outbreak of tuberculosis on a deer farm. Journal of the Japan Veterinary Medical Association 47: 433-436. 